IGPG-06/6-3

hep-th/0606232

\title{
Quantum Gravity and Higher Curvature Actions
}

\author{
Martin Bojowald ${ }^{1 *}$ and Aureliano Skirzewski ${ }^{2 \dagger}$ \\ ${ }^{1}$ Institute for Gravitational Physics and Geometry, \\ The Pennsylvania State University, \\ 104 Davey Lab, University Park, PA 16802, USA \\ ${ }^{2}$ Max-Planck-Institut für Gravitationsphysik, Albert-Einstein-Institut, \\ Am Mühlenberg 1, D-14476 Potsdam, Germany
}

\begin{abstract}
Effective equations are often useful to extract physical information from quantum theories without having to face all technical and conceptual difficulties. One can then describe aspects of the quantum system by equations of classical type, which correct the classical equations by modified coefficients and higher derivative terms. In gravity, for instance, one expects terms with higher powers of curvature. Such higher derivative formulations are discussed here with an emphasis on the role of degrees of freedom and on differences between Lagrangian and Hamiltonian treatments. A general scheme is then provided which allows one to compute effective equations perturbatively in a Hamiltonian formalism. Here, one can expand effective equations around any quantum state and not just a perturbative vacuum. This is particularly useful in situations of quantum gravity or cosmology where perturbations only around vacuum states would be too restrictive. The discussion also demonstrates the number of free parameters expected in effective equations, used to determine the physical situation being approximated, as well as the role of classical symmetries such as Lorentz transformation properties in effective equations. An appendix collects information on effective correction terms expected from loop quantum gravity and string theory.
\end{abstract}

\section{Introduction}

Quantum theories are usually full of considerable technical and conceptual difficulties. Even in mechanical systems which classically have only a finite number of degrees of freedom one has to deal with partial differential equations for a wave function. Such equations are more complicated to solve than the ordinary differential equations of a classical mechanical

*e-mail address: bojowald@gravity.psu.edu

${ }^{\dagger}$ e-mail address: skirz@aei.mpg.de 
system, and for their solutions one faces the usual interpretational issues. In quantum field theory the situation is correspondingly more complex since even classical field theories have infinitely many degrees of freedom. One is then dealing with some kind of functional differential equations in quantum field theory. This certainly applies also to gravity which is a field theory, unless one uses cosmological minisuperspace models with only a finite number of degrees of freedom. In the case of gravity, interpretational issues of the wave function related to gravity, such as the definition of observables or the problem of time, are even more severe.

It is therefore of considerable interest to find techniques which in certain regimes allow one to approximate the quantum system by a system of classical type, or effective system, by which we mean a system whose dynamical laws are of the same mathematical class as those of a corresponding classical system. For instance, an effective system of quantum mechanics would describe some regime of a quantum mechanical system by ordinary differential equations. This can be thought of as describing the motion of a wave packet by ordinary differential equations for the peak position rather than a partial differential equation for the whole wave function. In semiclassical regimes, effective equations should then be close to the classical ones, up to corrections of order $\hbar$. More generally, however, there can be other regimes where the classical equations are not valid at all, but some other equations of classical type still suffice. This is, for instance, the case if a wave packet spreads and deforms and these deformations back-react on the motion of the peak. Quantum properties are then important, but there could well be a finite number of parameters describing the wave packet well enough to justify the use of ordinary differential equations. An effective description is thus much more general than a semiclassical one.

A method of widespread use in particular in quantum field theory is that of low energy effective actions [1, which allows one to describe the quantum system by an effective action amending the classical action by quantum corrections but leaving the classical structure mainly untouched. In many cases, in particular when one expands perturbatively around a free field theory or a harmonic oscillator, such effective actions can be computed explicitly. The usual definition through a Legendre transform of the generating functional of irreducible $n$-point functions, however, looks very non-intuitive and seems tied to the situation of perturbative quantum field theory. Superficially, it looks unrelated to the above picture of traveling wave packets, and it is of deceptive uniqueness: there are usually no free parameters in the low energy effective action while an effective system for a quantum system should depend on the initial form of the wave packet, such as its initial spread, whose evolution is to be described effectively.

In this article we will discuss the relation between both types of effective systems from the point of view of a generalization of the usual effective action picture. This more general scheme has several advantages: (i) it does not restrict one to perturbative treatments and in fact allows all necessary freedom one may want to include in suitable initial states to perturb around, (ii) it applies to Hamiltonian methods as well and thus allows a comparison between effective pictures derived from canonical and covariant approaches to quantum field theory and (iii) by displaying all possible free parameters it shows the regimes where a particular effective action, including the standard low energy one, should or should 
not be applied. The last point has in particular implications for quantum gravity and cosmology, as we will discuss in the end. (Effective results from different quantum theories of gravity are collected in the Appendices.) We will also see that all crucial aspects of an effective action are already present for systems of finitely many degrees of freedom such as cosmological models in the context of gravity. It is thus sufficient for most purposes to use simplifications realized in such models, while field theoretical degrees of freedom increase the complexity considerably but do not add many distinctive properties.

\section{Higher derivatives}

Effective actions usually contain terms of the classical action with quantum corrections in the coefficients such as mass renormalization or corrections in an effective potential. An example is the effective action [2]

$\Gamma_{\text {eff }}[q]=\int \mathrm{d} t\left[\left(m+\frac{\hbar U^{\prime \prime \prime}(q)^{2}}{32 m^{2}\left(\omega^{2}+\frac{U^{\prime \prime}(q)}{m}\right)^{\frac{5}{2}}}\right) \frac{\dot{q}^{2}}{2}-\frac{1}{2} m \omega^{2} q^{2}-U(q)-\frac{\hbar \omega}{2}\left(1+\frac{U^{\prime \prime}(q)}{m \omega^{2}}\right)^{\frac{1}{2}}\right]$

for an an-harmonic oscillator with classical potential $\frac{1}{2} m \omega^{2} q^{2}+U(q)$. In addition, there will also be higher time derivative terms of the configuration variables in an expansion of slowly varying variables. This is often taken as an indication that there are additional degrees of freedom, albeit still finitely many ones, since more initial values have to be specified including higher derivatives in time of the classical variables. This appears to be along the lines sketched in the Introduction since a quantum system does have more degrees of freedom than a classical one. One needs an infinite number of parameters to specify a quantum state compared to finitely many ones in classical mechanics. Thus, in an approximation one has to include additional parameters to bridge the gap, bringing one in a possible full series summation of all higher derivative terms to an infinite number.

\subsection{Perturbation}

While this is true for any given higher derivative action, one has to be more careful in a perturbative scheme in which the higher derivative terms arise here. The highest order term in equations of motion following from such an effective action will always be multiplied by a power of the perturbation parameter such as $\hbar$. The unperturbed classical system is thus a singular point of the equations from a mathematical point of view [3]. Thus, most solutions will diverge when the perturbation parameter goes to zero, which should clearly be avoided for any solution one would trust within the perturbative scheme. Keeping solutions which are not analytic at zero perturbation parameter would violate the approximation in which effective equations have been derived. 
As an example, ${ }^{1}$ let us consider the field theory action

$$
S[\psi]=-\frac{1}{2} \int\left(\psi\left(\square+\epsilon \square^{2}\right) \psi+m^{2} \psi^{2}\right) \mathrm{d}^{3} x \mathrm{~d} t
$$

of a scalar field $\psi$ which has some higher derivative correction in a perturbation parameter $\epsilon$. The field equation is

$$
-\left(\square+\epsilon \square^{2}\right) \psi=m^{2} \psi
$$

with highest order term multiplied by $\epsilon$. The unperturbed theory $(\epsilon=0)$ is thus of lower order than the perturbed one and has less independent solutions.

In any such situation, there is a mismatch between unperturbed and perturbed solutions, and most solutions to the perturbed equations must be non-analytical in the perturbation parameter. For such solutions, higher order terms contribute uncontrolled corrections such that they have to be discarded in a perturbative treatment. Keeping them is inconsistent within the perturbative analysis. Only analytical solutions are to be retained, which do exist in the right number being the same as the number of unperturbed solutions [5]. In our example, for instance, we can look for plane wave solutions $\psi(x, t)=$ $\exp (i(E t-k x))$ which have to fulfill the dispersion relation $E^{2}=k^{2}-\frac{1}{2} \epsilon^{-1} \pm \frac{1}{2} \epsilon^{-1} \sqrt{1+4 \epsilon m^{2}}$. For $\epsilon \ll m^{-2}$, we can expand $E^{2}=k^{2}-\frac{1}{2} \epsilon^{-1}\left(1 \mp\left(1+2 \epsilon m^{2}-2 \epsilon^{2} m^{4}\right)\right)+O\left(\epsilon^{2}\right)$, clearly showing the existence of two analytical solutions in $\epsilon$, for which the unperturbed perturbation relation is just corrected by terms of the order $\epsilon, E^{2}=k^{2}+m^{2}-\epsilon m^{4}+O\left(\epsilon^{2}\right)$, and two non-analytical ones which have to be discarded.

There are thus no additional degrees of freedom in the sense of solutions, although corrections in solutions also come from higher derivative terms even in solutions analytical in the perturbation parameter. Thus, higher derivative effective actions, when treated consistently in a perturbative scheme, provide additional "quantum" degrees of freedom only implicitly. The discussion also implies that a description by an effective action is usually more complicated than it appears because one not only has to solve the equations of motion but also pick the correct ones which are analytical in the perturbation parameter 3]. This can be particularly difficult to do in numerical studies. Taking general solutions at face value, on the other hand, is misleading since this contains redundant, non-physical degrees of freedom.

\subsection{Hamiltonian picture}

The Hamiltonian picture of effective actions looks quite different from the Lagrangian viewpoint, although as usually both pictures are in the end equivalent. Degrees of freedom in a Hamiltonian formulation are given by coordinates and momenta, which are fixed from the outset independently of the Hamiltonian as a function of phase space variables but not their time derivatives. This is different from the Lagrangian which is a functional of the configuration variables as functions of time and thus determines what the independent degrees of freedom are. If one were to perturb a Hamiltonian theory in its given variables

\footnotetext{
${ }^{1}$ See also [4] for further discussion of this example.
} 
one would thus obtain correction terms, but no additional degrees of freedom, not even implicitly.

In fact, the process of expanding perturbatively does not commute with the Legendre transformation if higher time derivatives arise [4]. If we just expand a Hamiltonian in its classical variables we obtain a theory different from that obtained by expanding the corresponding action in a higher derivative expansion and then performing the Legendre transformation to a Hamiltonian picture. This is because a higher derivative action superficially introduces new degrees of freedom corresponding to higher derivatives and their momenta, which will then also occur in the Hamiltonian picture. Starting already at the Hamiltonian level, on the other hand, there is no obvious way to obtain new degrees of freedom.

To continue with our example, we have momenta $\pi_{\psi}=\dot{\psi}-2 \epsilon \Delta \dot{\psi}+\epsilon \mathrm{d}^{3} \psi / \mathrm{d} t^{3}$ and $\pi_{\dot{\psi}}=-\epsilon \ddot{\psi}$ conjugate to the independent configuration variables $\psi$ and $\dot{\psi}$. Also here, $\epsilon$ appears as a coefficient such that, when we invert the relations to replace $\ddot{\psi}$ and $\mathrm{d}^{3} \psi / \mathrm{d} t^{3}$ in the Hamiltonian, we divide by $\epsilon$ and the Hamiltonian

$$
H=\int \mathrm{d}^{3} x\left(-\frac{1}{2} \dot{\psi}^{2}+\dot{\psi} \pi_{\psi}-\frac{1}{2} \psi \Delta \psi+\frac{1}{2} m^{2} \psi^{2}+\epsilon\left(\dot{\psi} \Delta \dot{\psi}+\psi \Delta^{2} \psi / 2\right)-\frac{1}{2} \epsilon^{-1} \pi_{\dot{\psi}}^{2}\right)
$$

of the higher derivative action is not analytic in the perturbation parameter. Not all these terms could have been obtained from a perturbative treatment at the Hamiltonian level. In such a case, we could have derived at most the perturbative terms in (4) which are analytic in $\epsilon$. The analytic part of the Hamiltonian implies the standard momenta $\pi_{\psi}=\dot{\psi}+O(\epsilon)$ to leading order: without additional degrees of freedom, $\dot{\psi}$ and $\pi_{\psi}$ are not independent, $\delta \dot{\psi} / \delta \pi_{\psi} \neq 0$, such that the Hamiltonian equation of motion from the part of (4I) analytic in $\epsilon$,

$$
\dot{\psi}=\delta H / \delta \pi_{\psi}=-\dot{\psi} \partial \dot{\psi} / \partial \pi_{\psi}+\dot{\psi}+\pi_{\psi} \partial \dot{\psi} / \pi_{\psi}+2 \epsilon \Delta \dot{\psi} \partial \dot{\psi} / \partial \pi_{\psi}+O\left(\epsilon^{2}\right),
$$

requires $\pi_{\psi}=\dot{\psi}-2 \epsilon \Delta \dot{\psi}$. This results in the perturbative Hamiltonian

$$
H=\frac{1}{2}\left(\pi_{\psi}^{2}-\psi \Delta \psi+m^{2} \psi^{2}+\epsilon\left(2 \pi_{\psi} \Delta \pi_{\psi}+\psi \Delta^{2} \psi\right)\right) .
$$

In second order form, we obtain the equation of motion

$$
\ddot{\psi}=\Delta \psi-m^{2} \psi+\epsilon\left(\Delta^{2} \psi-2 m^{2} \Delta \psi\right)+O\left(\epsilon^{2}\right)
$$

which is different from the Lagrangian one (3).

\section{Quantum degrees of freedom}

The example illustrates that the Hamiltonian picture requires more refined methods to derive corrections mediated through higher derivatives or some other kind of new degrees of freedom not present in the classical system. As described in the introduction, this can be possible by describing a trajectory of semiclassical wave packets by effective equations. 
For a mechanical system of a single degree of freedom, we have classical variables $q=\langle\hat{q}\rangle$, $p=\langle\hat{p}\rangle$ associated with expectation values of quantum operators. This allows us to make an identification between some degrees of freedom of the quantum theory and the classical ones. A wave packet, however, has more information than just expectation values of basic operators because we have, e.g., $\left\langle\hat{q}^{n}\right\rangle \neq\langle\hat{q}\rangle^{n}$. Unlike the classical situation where $q^{n}$ would directly be obtained by taking a power of $q$, the expectation value of $\hat{q}^{n}$ has additional information not contained in $\langle\hat{q}\rangle^{n}$. This additional information can be captured in quantum variables 6 ]

$$
G^{a, n}:=\left\langle(\hat{q}-\langle\hat{q}\rangle)^{n-a}(\hat{p}-\langle\hat{p}\rangle)^{a}\right\rangle_{\mathrm{Weyl}} \quad, \quad a=0,1, \ldots, n
$$

which are independent of the classical ones (the subscript "Weyl" denoting symmetric ordering of the operators). In fact, one can define a symplectic structure, using the imaginary part of the inner product, for these variables such that quantum mechanics is formulated on an $\infty$-dimensional phase space. If we label states $|\psi\rangle=\sum_{j} c_{j}\left|\psi_{j}\right\rangle$ by expansion coefficients $c_{j}$ in some orthonormal basis $\left|\psi_{j}\right\rangle$, such that the inner product between two states $|\psi\rangle$ and $|\phi\rangle=\sum_{j} d_{j}\left|\psi_{j}\right\rangle$ is $\langle\psi, \phi\rangle=\sum_{j} \bar{c}_{j} d_{j}$, we can use real and imaginary parts of the $c_{j}$ as real coordinates on the Hilbert space and define the symplectic structure [7]

$$
\Omega\left(\delta c_{j}, \delta d_{k}\right):=2 \hbar \operatorname{Im}\langle\psi, \phi\rangle=2 \hbar \sum_{j}\left(\operatorname{Re} \delta c_{j} \operatorname{Im} \delta d_{j}-\operatorname{Im} \delta c_{j} \operatorname{Re} \delta d_{j}\right)
$$

evaluated in two vectors with components $\delta c_{j}$ and $\delta d_{k}$. From this, we read off the Poisson brackets $\left\{\operatorname{Re} c_{j}, \operatorname{Im} c_{k}\right\}=\frac{1}{2 \hbar} \delta_{j k}$, and vanishing brackets between real and imaginary parts, respectively. While the Poisson relations are most easily determined for expansion coefficients $c_{j}$, these variables are not very suitable to be split into classical and quantum parts. We will therefore use below the variables $q, p$ and $G^{a, n}$ and also state their Poisson relations there.

In this picture, the Schrödinger equation for $|\psi\rangle$ is equivalent to Hamiltonian equations of motion with the above Poisson brackets and Hamiltonian function given by the expectation value $H_{Q}(\psi)=\langle\psi, \hat{H} \psi\rangle$, seen as a function on the Hilbert space. This follows easily if we choose the orthonormal basis above to be given by eigenstates of the Hamiltonian operator, $\hat{H}\left|\psi_{j}\right\rangle=E_{j}\left|\psi_{j}\right\rangle$. Then, $H_{Q}\left(c_{j}\right)=\sum_{j} E_{j}\left|c_{j}\right|^{2}=\sum_{j} E_{j}\left(\left(\operatorname{Re} c_{j}\right)^{2}+\left(\operatorname{Im} c_{j}\right)^{2}\right)$ and we have equations of motion

$$
\frac{\mathrm{d}}{\mathrm{d} t} \operatorname{Re} c_{j}=\left\{\operatorname{Re} c_{j}, H_{Q}\right\}=\frac{E_{j}}{\hbar} \operatorname{Im} c_{j} \quad, \quad \frac{\mathrm{d}}{\mathrm{d} t} \operatorname{Im} c_{j}=\left\{\operatorname{Im} c_{j}, H_{Q}\right\}=-\frac{E_{j}}{\hbar} \operatorname{Re} c_{j}
$$

or $\dot{c}_{j}=-i \hbar^{-1} E_{j} c_{j}$ with solution $c_{j}(t)=\exp \left(-i \hbar^{-1} E_{j} t\right)$ which is equivalent to the solutions of the Schrödinger equation.

This geometrical picture of quantum mechanics [7] has been used for semiclassical definitions in [8, 9], and in [6] for developing the point of view of effective systems used here. $^{2}$ There are thus truly infinitely many quantum degrees of freedom, although their

\footnotetext{
${ }^{2}$ Some of these ideas look related to ingredients of Ehrenfest theorems or the WKB approximation. However, the geometrical formulation allows much tighter control over all the correction terms to classical behavior. For an-harmonic oscillators discussed later, for instance, the WKB approximation agrees with effective action results only to first order in the anharmonicity parameter [10].
} 
role as higher derivatives of classical variables or something else can only be found after studying dynamical equations.

In fact, the quantum variables are dynamical and in general back-react on the classical variables: the $G^{a, n}$ change if a wave packet spreads and deforms. For a quadratic Hamiltonian in canonical variables, we can simply use relations such as $\left\langle\hat{q}^{2}\right\rangle=\langle\hat{q}\rangle^{2}+G^{0,2}$ which imply that terms containing the quantum variables are just added to the classical Hamiltonian. This can give zero point energy contributions, but since no products between classical and quantum variables occur, there are no coupling terms. If the classical Hamiltonian is not quadratic in the canonical variables, on the other hand, coupling terms between the quantum and classical variables occur by expanding the expectation value of the Hamiltonian operator in a general state. To make this explicit, we write the quantum Hamiltonian as

$$
\begin{aligned}
H_{Q} & =\langle H(\hat{q}, \hat{p})\rangle_{\mathrm{Weyl}}=\langle H(q+(\hat{q}-q), p+(\hat{p}-p))\rangle_{\mathrm{Weyl}} \\
& =\sum_{n=0}^{\infty} \sum_{a=0}^{n} \frac{1}{n !}\left(\begin{array}{c}
n \\
a
\end{array}\right) \frac{\partial^{n} H(q, p)}{\partial p^{a} \partial q^{n-a}} G^{a, n}
\end{aligned}
$$

where from now on $q=\langle\hat{q}\rangle$ and $p=\langle\hat{p}\rangle$. Since $G^{a, 0}=1$ and $G^{a, 1}=0$ by definition, the quantum variables appear starting at second order $G^{a, 2}$ with coefficients proportional to $\partial^{2} H(q, p) / \partial p^{a} \partial q^{2-a}$. These are constants for a quadratic potential, where all higher coefficients vanish, but do depend on the classical variables for Hamiltonians with nonharmonic potentials or non-standard kinetic terms. In the latter cases, thus, coupling terms between classical and quantum variables arise.

For the dynamical behavior of classical and quantum variables we need to compute the Hamiltonian equations of motion

$$
\dot{q}=\left\{q, H_{Q}\right\} \quad, \quad \dot{p}=\left\{p, H_{Q}\right\} \quad, \quad \dot{G}^{a, n}=\left\{G^{a, n}, H_{Q}\right\} .
$$

For this, we need to know the symplectic structure [6] which is given by the classical Poisson brackets $\{q, p\}=1$ together with $\left\{q, G^{a, n}\right\}=0=\left\{p, G^{a, n}\right\}$ and

$$
\begin{gathered}
\left\{G^{a, n}, G^{b, m}\right\}=\sum_{r}\left[\left(\frac{\hbar}{2}\right)^{2 r} K[a, b, m, n, r] G^{a+b-2 r-1, m+n-4 r-2}\right] \\
-b(n-a) G^{a, n-1} G^{b-1, m-1}+a(m-b) G^{b, m-1} G^{a-1, n-1}
\end{gathered}
$$

where

$$
K[a, b, m, n, r]=\sum_{0 \leq f \leq 2 r+1}(-)^{r+f}(f !(2 r+1-f) !)^{-1}\left(\begin{array}{l}
a \\
f
\end{array}\right)\left(\begin{array}{l}
n-a \\
2 r+1-f
\end{array}\right)\left(\begin{array}{l}
b \\
f
\end{array}\right)\left(\begin{array}{l}
m-b \\
2 r+1-f
\end{array}\right) .
$$

If we rescale our quantum variables by $\tilde{G}^{a, n}=\hbar^{-n / 2}(m \omega)^{n / 2-a} G^{a, n}$ to make them dimensionless and compute the equations for an an-harmonic oscillator with classical Hamiltonian $H=\frac{1}{2 m} p^{2}+\frac{1}{2} m \omega^{2} q^{2}+U(q)$, we obtain the quantum Hamiltonian

$$
H_{Q}=\frac{1}{2 m} p^{2}+\frac{1}{2} m \omega^{2} q^{2}+U(q)+\frac{\hbar \omega}{2}\left(\tilde{G}^{0,2}+\tilde{G}^{2,2}\right)+\sum_{n} \frac{1}{n !}\left(\frac{\hbar}{m \omega}\right)^{n / 2} U^{(n)}(q) \tilde{G}^{0, n}
$$


as an expansion in $\hbar$ which clearly shows the coupling terms between classical and quantum variables coming from a non-quadratic potential. With the above Poisson brackets, $H_{Q}$ generates equations of motion

$$
\begin{aligned}
\dot{q}= & \frac{p}{m} \\
\dot{p}= & -m \omega^{2} q-U^{\prime}(q)-\sum_{n} \frac{1}{n !}\left(\frac{\hbar}{m \omega}\right)^{n / 2} U^{(n+1)}(q) \tilde{G}^{0, n} \\
\dot{\tilde{G}}^{a, n}= & -a \omega \tilde{G}^{a-1, n}+(n-a) \omega \tilde{G}^{a+1, n}-a \frac{U^{\prime \prime}(q)}{m \omega} \tilde{G}^{a-1, n} \\
& +\frac{\sqrt{\hbar} a U^{\prime \prime \prime}(q)}{2(m \omega)^{\frac{3}{2}}} \tilde{G}^{a-1, n-1} \tilde{G}^{0,2}+\frac{\hbar a U^{\prime \prime \prime \prime}(q)}{3 !(m \omega)^{2}} \tilde{G}^{a-1, n-1} \tilde{G}^{0,3} \\
& -\frac{a}{2}\left(\frac{\sqrt{\hbar} U^{\prime \prime \prime}(q)}{(m \omega)^{\frac{3}{2}}} \tilde{G}^{a-1, n+1}+\frac{\hbar U^{\prime \prime \prime \prime}(q)}{3(m \omega)^{2}} \tilde{G}^{a-1, n+2}\right) \\
& +\frac{a(a-1)(a-2)}{24}\left(\frac{\sqrt{\hbar} U^{\prime \prime \prime}(q)}{(m \omega)^{\frac{3}{2}}} G^{a-3, n-3}+\frac{\hbar U^{\prime \prime \prime \prime}(q)}{(m \omega)^{2}} \tilde{G}^{a-3, n-2}\right)+\cdots
\end{aligned}
$$

These infinitely many coupled ordinary differential equations are equivalent to the Schrödinger equation and in general not easier to solve. However, this set of equations is much more suitable for splitting the dynamics in classical effective equations and equations for quantum degrees of freedom. We also note that such a system of infinitely many differential equations requires infinitely many parameters as initial conditions. Although they are not completely arbitrary, as the quantum variables have to satisfy uncertainty relations such

as $G^{0,2} G^{2,2} \geq \frac{\hbar^{2}}{4}+\left(G^{1,2}\right)^{2}$, fixing these values will turn out to be one of the crucial parts of deriving effective equations.

\subsection{Example: Harmonic oscillator}

The Hamiltonian of the harmonic oscillator is quadratic in the canonical variables and there are thus no coupling terms between classical and quantum variables. This conforms with the well-known fact that there are dynamical coherent states for the harmonic oscillator which do neither spread nor deform while following the classical trajectories exactly. At the quantum level, we have Hamiltonian equations of motion

$$
\begin{aligned}
\dot{p} & =\left\{p, H_{Q}\right\}=-m \omega^{2} q \\
\dot{q} & =\left\{q, H_{Q}\right\}=\frac{1}{m} p \\
\dot{G}^{a, n} & =\left\{G^{a, n}, H_{Q}\right\}=\frac{1}{m}(n-a) G^{a+1, n}-m \omega^{2} a G^{a-1, n}
\end{aligned}
$$


which indeed decouples to an infinite set of finitely many coupled differential equations. Moreover, one can see that constant solutions for the quantum variables exist,

$$
G^{a, n}=2^{-n} \hbar^{1 / 2}(m \omega)^{a-n / 2} \frac{a !}{(a / 2) !} \frac{(n-a) !}{((n-a) / 2) !}
$$

saturating the uncertainty relations. These are exactly the solutions corresponding to coherent states. Since quantum variables do not appear in the above equations of motion for classical variables, there is no need to introduce new effective equations. Indeed, effective actions for "free" theories such as the harmonic oscillator are always identical to the classical action. Nevertheless, even in this simple example we can already see the generality of the effective equation scheme discussed here: We can just as well choose non-constant solutions for $G^{a, n}$ which then change cyclically along the classical orbit. This describes wave packets which deform periodically while following classical peak positions, a behavior which can be interpreted as semiclassical, just as the case of constant $G^{a, n}$, provided that the $G^{a, n}$ do not change too rapidly.

\subsection{An-harmonic oscillator}

The situation is more interesting for non-quadratic classical Hamiltonians because for them coupling terms between classical and quantum variables appear. All infinitely many differential equations for the classical and quantum variables are then coupled and $(q, p)$ are affected by the motion of $G^{a, n}$ in non-trivial ways. This describes the back-reaction of spreading and deformations of the wave packet on their peak positions.

For practical purposes, this set of infinitely many coupled equations must be truncated to a finite set in suitable approximations, such as the adiabatic approximation in quantum variables. In this approximation, equations for $G^{a, n}$ can be solved perturbatively in $\hbar$ and in the adiabatic expansion. The latter is an expansion in a parameter $\lambda$ formally introduced in the calculation, but in the end set to $\lambda=1$. Derivatives with respect to time in equations of motion are first re-scaled as $\frac{\mathrm{d}}{\mathrm{d} t} \rightarrow \lambda \frac{\mathrm{d}}{\mathrm{d} t}$. Moreover, slowly changing variables for which the adiabatic approximation is done are expanded in a series in $\lambda$ as well. We expand only the quantum variables $G^{a, n}=\sum_{e} G_{e}^{a, n} \lambda^{e}$ in this manner, meaning that their change in time is adiabatic, but keep the evolution of classical variables free. After inserting this in the equations of motion and expanding in $\lambda$, one obtains equations for all coefficients $G_{e}^{a, n}$ at different orders of the adiabatic expansion. ${ }^{3}$ The equations of motion $\dot{G}^{a, n}=\left\{G^{a, n}, H_{Q}\right\}$ for quantum variables then imply $\dot{G}_{e-1}^{a, n}=\left\{G_{e}^{a, n}, H_{Q}\right\}$. In addition to the adiabatic approximation there is also a semiclassical expansion in powers of $\hbar$. To obtain effective equations to the order relevant for (11), it turns out that one has to calculate the first order in $\hbar$ and go to second order in $\lambda$ for $G^{a, 2}$.

We can now use the Poisson relations

$$
\left\{G^{a, n}, G^{0,2}\right\}=-2 a G^{a-1, n} \quad \text { and } \quad\left\{G^{a, n}, G^{2,2}\right\}=2(n-a) G^{a+1, n}
$$

\footnotetext{
${ }^{3}$ For more details on the following calculations and underlying definitions, see [6].
} 
giving equations

$$
0=\left\{G_{0}^{a, n}, H_{Q}\right\}=\omega\left((n-a) G_{0}^{a+1, n}-a\left(1+\frac{U^{\prime \prime}(q)}{m \omega^{2}}\right) G_{0}^{a-1, n}\right)
$$

at zeroth order in $\lambda$ used for all $n$,

$$
\dot{G}_{0}^{a, n}=\left\{G_{1}^{a, n}, H_{Q}\right\}=\omega\left((n-a) G_{1}^{a+1, n}-a\left(1+\frac{U^{\prime \prime}(q)}{m \omega^{2}}\right) G_{1}^{a-1, n}\right)
$$

to first order in $\lambda$ used for all $n$, and

$$
G_{2}^{2,2}-\left(1+\frac{U^{\prime \prime}(q)}{m \omega^{2}}\right) G_{2}^{0,2}=\frac{1}{\omega} \dot{G}_{1}^{0,2}=\frac{1}{2 \omega^{2}} \ddot{G}_{0}^{0,2}
$$

to second order in $\lambda$ at $n=2$.

The general solution of (18) is

$$
G_{0}^{a, n}=\left(\begin{array}{c}
n / 2 \\
a / 2
\end{array}\right)\left(\begin{array}{c}
n \\
a
\end{array}\right)^{-1}\left(1+\frac{U^{\prime \prime}(q)}{m \omega^{2}}\right)^{a / 2} G_{0}^{0, n}
$$

for even $n$ and $a$ and zero whenever $a$ and/or $n$ are odd. This still leaves the value of $G_{0}^{0, n}$ free, which will be fixed shortly. The first order equation (19) then implies

$$
\begin{aligned}
& \frac{1}{\omega} \sum_{a}\left(\begin{array}{c}
n / 2 \\
a / 2
\end{array}\right)\left(1+\frac{U^{\prime \prime}(q)}{m \omega^{2}}\right)^{(n-a) / 2} \dot{G}_{0}^{a, n} \\
= & \sum_{a \text { even }}\left(\begin{array}{c}
n / 2 \\
a / 2
\end{array}\right)\left(1+\frac{U^{\prime \prime}(q)}{m \omega^{2}}\right)^{(n-a) / 2}\left((n-a) G_{1}^{a+1, n}-a\left(1+\frac{U^{\prime \prime}(q)}{m \omega^{2}}\right) G_{1}^{a-1, n}\right) \\
= & 0
\end{aligned}
$$

which can be seen by shifting $a \rightarrow a-2$ in the first term of the right hand side. This imposes a constraint on $G_{0}^{0, n}$ solved by $G_{0}^{0, n}=C_{n}\left(1+\frac{U^{\prime \prime}(q)}{m \omega^{2}}\right)^{-n / 4}$. The remaining constants $C_{n}$ can be fixed to $C_{n}=\frac{n !}{2^{n}(n / 2) !}$ by requiring that the limit $U(q) \rightarrow 0$ reproduces the quantum variables (17) of coherent states of the harmonic oscillator. This means that we require the perturbative vacuum of the quantum theory to be reproduced in the effective system. Therefore,

$$
G_{0}^{a, n}=\frac{(n-a) ! a !}{2^{n}((n-a) / 2) !(a / 2) !}\left(1+\frac{U^{\prime \prime}(q)}{m \omega^{2}}\right)^{\frac{2 a-n}{4}} .
$$

From the first order corrections $G_{1}^{a, n}$, we will only need solutions for $n=2$ which follow directly from (19) with $a=0$ as $G_{1}^{1,2}=\frac{1}{2 \omega} \dot{G}_{0}^{0,2}$. The second order equation (20) 
again leaves free parameters in the general solution to be fixed by the next, third order: $\left(1+U^{\prime \prime}(q) /\left(m \omega^{2}\right)\right) \dot{G}_{2}^{0,2}+\dot{G}_{2}^{2,2}=0$ as before. From this, the solution to the system is

$$
\begin{aligned}
G_{2}^{0,2} & =-\frac{2}{\omega^{2}}\left(G_{0}^{0,2}\right)^{\frac{5}{2}}\left(\left(G_{0}^{0,2}\right)^{\frac{1}{2}}\right)^{\cdot} \\
& =\frac{\left(1+\frac{U^{\prime \prime}(q)}{m \omega^{2}}\right)^{-\frac{7}{2}}}{4 \omega^{2}}\left(\left(1+\frac{U^{\prime \prime}(q)}{m \omega^{2}}\right) \frac{U^{\prime \prime \prime}(q) \ddot{q}+U^{\prime \prime \prime \prime}(q) \dot{q}^{2}}{4 m \omega^{2}}-5\left(\frac{U^{\prime \prime \prime}(q) \dot{q}}{4 m \omega^{2}}\right)^{2}\right) .
\end{aligned}
$$

Finally, putting our approximate expressions for the quantum variables back into the equations

$$
\begin{aligned}
\dot{q} & =m^{-1} p \\
\dot{p} & =-m \omega^{2} q-U^{\prime}(q)-\sum_{n} \frac{1}{n !}\left(m^{-1} \omega^{-1} \hbar\right)^{n / 2} U^{(n+1)}(q) G^{0, n}
\end{aligned}
$$

for the classical variables and writing them as a second order equation for $q$, we obtain

$$
\begin{aligned}
& \left(m+\frac{\lambda^{2} \hbar U^{\prime \prime \prime}(q)^{2}}{32 m^{2} \omega^{5}\left(1+\frac{U^{\prime \prime}(q)}{m \omega^{2}}\right)^{\frac{5}{2}}}\right) \ddot{q} \\
& +\frac{\lambda^{2} \hbar \dot{q}^{2}\left(4 m \omega^{2} U^{\prime \prime \prime}(q) U^{\prime \prime \prime \prime}(q)\left(1+\frac{U^{\prime \prime}(q)}{m \omega^{2}}\right)-5 U^{\prime \prime \prime}(q)^{3}\right)}{128 m^{3} \omega^{7}\left(1+\frac{U^{\prime \prime}(q)}{m \omega^{2}}\right)^{\frac{7}{2}}} \\
& +m \omega^{2} q+U^{\prime}(q)+\frac{\hbar U^{\prime \prime \prime}(q)}{4 m \omega\left(1+\frac{U^{\prime \prime}(q)}{m \omega^{2}}\right)^{\frac{1}{2}}}=0 .
\end{aligned}
$$

After setting $\lambda=1$, we finally have our effective equations to first order in $\hbar$, which agree with the equations of motion determined by (11). Thus, the methods described here provide a generalized derivation of effective equations, and in particular a Hamiltonian picture.

\subsection{General effective systems}

Since the main difference for dynamical purposes between classical and quantum mechanical systems lies in the infinite dimensionality of a quantum system compared to the finite dimensionality of a classical mechanical one, the main aim of any effective approximation is a truncation of the quantum dynamics to an effective one on a finite dimensional subspace. Moreover, almost all of the infinitely many integration constants, corresponding to the shape of an initial quantum state, required for the full quantum system have to be fixed by some means. In the above procedure for an an-harmonic oscillator, the adiabatic approximation together with an $\hbar$-expansion required only a finite number of $G^{a, n}$ at each order, resulting in a truncation of the quantum system to an effective one. The 
integration constants such as $C_{n}$ were fixed by relating them to properties of harmonic oscillator coherent states. But even in this case, other choices are possible and will result in different effective equations describing states which do not saturate uncertainty relations or are squeezed. This shows that effective equations in general terms will never be unique, in contrast to the low energy effective action obtained by perturbing around the vacuum state, but will occur in parameterized form to describe different physically relevant sectors. The low energy effective action is not suitable for any such situation that may arise because one cannot describe arbitrary states as perturbations around a ground state.

The example of an-harmonic oscillators also elucidates the role of higher derivatives and their potential relation to quantum degrees of freedom. In the geometrical picture, there is a consistent way to introduce additional degrees of freedom by keeping some of the $G^{a, n}$ as independent variables whose solutions are not inserted into the equations of classical variables. Only the remaining quantum variables are then solved approximately and inserted in equations for classical variables as well as those of the quantum variables we kept. This gives a higher dimensional effective system with new, non-classical degrees of freedom. While it may then be possible in some cases to view those additional degrees of freedom as higher derivatives of the classical ones, after making explicit use of equations of motion, this will not be the same as higher derivatives in a higher derivative Lagrangian. In the latter case, higher derivatives as degrees of freedom are only implicit as discussed earlier, while keeping additional $G^{a, n}$ as true independent degrees of freedom is consistent in the perturbation scheme. Implicit effects of higher derivative terms in a low energy effective action correspond, rather, to using higher orders in an adiabatic approximation of the quantum variables following the same procedure as before, i.e. using only $q$ and $p$ in effective equations.

An example where one has to use some quantum variables as independent degrees of freedom is the free particle. The adiabatic approximation is not consistent in this case, as one can see from the fact that the effective action (11) diverges for a potential $U(q)=-\frac{1}{2} m \omega^{2} q^{2}$ which cancels the harmonic contribution exactly. This is analogous to infrared problems in the massless limit of quantum field theory. In this case, the spreading of wave packets is too strong to allow an adiabatic approximation, but keeping the spread as one of the independent quantum variables allows a well-defined effective formulation [6]. A more interesting example is cosmological structure formation where keeping quantum variables of order two in effective equations shows how quantum fluctuations seed classical metric perturbations [12].

\subsection{Comparison}

For free theories such as the harmonic oscillator there is no difference between the standard low energy effective action and the general methods described here, because quantum variables do not couple to classical variables. Thus, although one can choose non-vacuum states to expand around and thereby obtain different dynamics for the quantum variables, this does not back-react on the dynamics of classical variables. There are other common properties such as the fact that low energy effective actions for non-quadratic theories arise 
from non-local objects in time, such that no truncation to finite order in higher derivatives can give exact results, which is related to the fact that there are infinitely many variables in a quantum theory and thus infinitely many coupled equations. In the same way as a local approximation of the low energy effective action can be done by performing a derivative expansion, the infinitely many quantum variables of general effective systems can be cut down to a finite number in an adiabatic approximation. Non-local features of the low-energy effective action occur through higher derivative terms, suggesting additional degrees of freedom which may be seen in analogy to the independent quantum variables. Here, both pictures are starting to become different because higher derivative actions in a perturbative formulation are more subtle, as discussed before, and do not obviously have additional degrees of freedom, while quantum variables are true degrees of freedom of a quantum theory. There is also a difference in the interpretation of variables entering effective equations: In the usual picture of low-energy effective actions, "classical" variables are related to non-diagonal matrix elements of operators which are not guaranteed to be real [11. In the general scheme of effective systems, on the other hand, classical variables are expectation values of the basic operators and thus always real.

Further differences refer to the general form of effective equations and their uniqueness. While the low energy effective action is by definition obtained by perturbing around the vacuum state, one can derive effective systems more generally for any state. Thus, while there are no free parameters in the low energy effective action which appears to be unique, initial conditions of the quantum variables describing spread and deformations of the initial state appear in general effective equations. In a situation where one has a unique way to fix the state, such as the harmonic oscillator ground state, one reproduces low energy results by the more general method. This comparison is summarized in Tab. 1.

\begin{tabular}{cc}
\hline low energy & general \\
\hline \hline free theories (harmonic oscillator) unchanged \\
\hline non-local & $\infty$-many coupled equations \\
\hline derivative expansion & adiabatic approximation \\
\hline higher derivatives & independent quantum variables \\
\hline "classical" variables related to & expectation values in \\
non-diagonal matrix elements & dynamical coherent states \\
\hline expansion around free theory & expansion possible around \\
& any state, such as squeezed ones \\
\hline "unique," free of parameters & free parameters from initial conditions \\
& of $G^{a, n}$ specify state to expand around \\
\hline usual effective action (11) for expansion around the harmonic oscillator vacuum \\
\hline
\end{tabular}

Table 1: Comparison between properties of low energy effective actions and general effective systems. 


\section{Implications for quantum gravity}

By embedding the standard procedure to arrive at low energy effective actions into a general scheme, we have seen that more general effective descriptions cannot be unique. They rather depend on parameters corresponding to the choice of a state to expand around. In low energy effective actions, this state is chosen to be the perturbative vacuum state which fixes the parameters, but this is not available for more general situations. The low energy effective action is in fact what is relevant for low energies and only small excitations out of the vacuum. But for applications in gravity, unless one is dealing with graviton scattering, the applicability of a low energy effective action is questionable. This is in particular true for quantum cosmology based on effective actions because the whole universe is far from a gravitational vacuum state, however this may be defined. For instance, in background dependent quantizations of gravity, the background metric is used to define the perturbative vacuum, resulting in higher curvature low energy effective actions. It is then sometimes tempting to use these effective actions for a general metric and study implications even in strong curvature regimes of black holes or cosmology far away from the original background. However, this is clearly outside the allowed range of validity of the approximation.

A quantum cosmological situation is not expected to be described by expanding around the vacuum state of a free field theory; an effective description should rather be obtained by expanding around a suitable initial state describing a semiclassical universe at large scales but being far from a vacuum state. Unlike the vacuum, such states are not unique but depend on several parameters. Then, also effective equations obtained by such an approximation depend on the same parameters: they appear as initial values for the quantum variables $G^{a, n}$ which, when solutions are inserted in the equations for classical variables, also enter the effective equations. Thus, a general effective description relevant for most purposes of gravity or cosmology cannot be as unique as an effective action obtained by perturbing around the vacuum. Parameters that will appear in general are not simply ambiguities of the formalism, but they have physical relevance as they describe properties such as the spread of an initial state. The dynamics will in general depend on the spread such that it must enter the effective equations. The choice of parameters thus can be fixed by physical considerations, just as the vacuum state chosen in low energy effective actions determines the initial spread and deformations.

This is the situation naturally encountered in background independent quantum theories of gravity where it is anyway more difficult to define a perturbative vacuum state. One would thus use a class of semiclassical states, parameterized in some way if no distinguished state is known, to compute expectation values of Hamiltonians. These parameters then appear in effective equations as they do even in mechanical systems. Some of those parameters also occur in effective actions derived from background dependent quantizations since one can sometimes use different backgrounds to perturb around. However, there are other, truly quantum parameters which are not taken into account by just changing the background, but which may be important physically.

Finally, while the vacuum state is Lorentz invariant and the corresponding effective action is covariant, a general state will not be preserved by a Lorentz transformation. This 
is in particular true for a semiclassical state corresponding to an expanding cosmology. Thus, effective equations relevant for quantum gravity or cosmology should not be expected to be Lorentz covariant. Performing a Lorentz transformation maps the chosen state to a different one, thereby changing the parameters appearing in effective equations. These equations, then, also change to a different set. (Sometimes, in particular when parameters come from different metric backgrounds, one can view a whole class of effective systems as one system invariant under Lorentz transformations, for which also background parameters change. But all these effective systems and the background parameters really come from different states which are then being transformed into each other.) Even if the original theory of quantum gravity for which an effective system is considered were covariant, the effective equations will not be. This comes about because one is forced to treat all the degrees of freedom entering the quantum system differently, by formulating effective equations for some of them but keeping the rest only in a parameterized way. This cannot be avoided because the whole point of an effective description is to cut down the infinite number of quantum variables to a finite set. Lorentz transformations at the quantum level, in general, mix all these parameters and cannot be constrained to act on just the variables kept for an effective system. It is then impossible to draw conclusions concerning Lorentz invariance or violation based on just one set of effective equations, but a wider view based on more general properties of effective systems can give indications for symmetries of a quantum theory of gravity.

\section{Acknowledgments}

We are grateful to Abhay Ashtekar and Jürg Käppeli for discussions. MB thanks the organizers of the 42nd Karpacz Winter School of Theoretical Physics "Current Mathematical Topics in Gravitation and Cosmology," Ladek, Poland, February 6-11, 2006 and in particular Andrzej Borowiec for an invitation to give a lecture course on which this text is based.

\section{A Loop quantum gravity and cosmology}

In these appendices we summarize the situation in the main current candidates for quantum theories of gravity, loop quantum gravity and string theory, from the point of view of effective actions and higher curvature terms. This presents one example each for the two different classes of background independent and background dependent quantizations. We provide more details on loop quantum gravity since this is the lesser known framework (which is also subject to the common misconception that effective correction terms would not arise here) but closer to the authors' expertise, and certainly not because there would be more results on effective actions from loop quantum gravity.

Loop quantum gravity is based on a canonical quantization such that space and time coordinates are treated differently. This is relevant for the appearance of higher deriva- 
tive terms because time-derivatives are replaced by momenta while space-derivatives are retained. Moreover, the loop quantization provides a discrete spatial structure such that difference operators rather than differential ones occur. It is thus easy to see higher spatial derivatives emerging for effective field equations, but for higher time derivatives one has to do more involved calculations using quantum variables as described before. Based on the presence of higher spatial derivatives but no higher time derivatives, possible Lorentz violations have been suggested [13, but as mentioned in Sec. 4] a final verdict has to await a more detailed analysis. This requires also higher time derivative corrections which have not been derived yet for any model of loop quantum gravity, but they can certainly arise along the lines of quantum variables described in the main text. To see why higher spatial derivatives must occur, we present a brief, mostly self-contained introduction to loop quantum gravity. More detailed reviews of these aspects are, e.g., [14.

\section{A.1 Canonical quantization}

Rather than using ADM variables ${ }^{4}$ as in a Wheeler-DeWitt quantization, loop quantum gravity uses Ashtekar variables [16, 17] where the field equations are easier to handle. The spatial metric is then replaced by a densitized triad $E_{i}^{a}$, such that $E_{i}^{a} E_{i}^{b}=q^{a b} \operatorname{det} q$, which also determines the spin connection $\Gamma_{a}^{i}$ compatible with $E_{i}^{a}$. The spin connection, in turn, together with extrinsic curvature components determines the connection $A_{a}^{i}=\Gamma_{a}^{i}+\gamma K_{a}^{i}$ (with Barbero-Immirzi parameter $0<\gamma \in \mathbb{R}$ [17, 18]) which is canonically conjugate to the densitized triad.

The Hamiltonian consists only of constraints due to general covariance, $H=G[\Lambda]+$ $D\left[N^{a}\right]+H[N]=0$, with the Gauss constraint $G[\Lambda]$ (generating triad rotations), the diffeomorphism constraint $D\left[N^{a}\right]$ (generating spatial diffeomorphisms) and the Hamiltonian constraint

$$
\begin{aligned}
H[N]= & \frac{1}{16 \pi G} \int_{\Sigma} \mathrm{d}^{3} x N|\operatorname{det} E|^{-1 / 2}\left(\epsilon_{i j k} F_{a b}^{i} E_{j}^{a} E_{k}^{b}\right. \\
& \left.-2\left(1+\gamma^{-2}\right)\left(A_{a}^{i}-\Gamma_{a}^{i}\right)\left(A_{b}^{j}-\Gamma_{b}^{j}\right) E_{i}^{[a} E_{j}^{b]}\right)
\end{aligned}
$$

which is the most complicated of the constraints. In this expression, $F_{a b}^{i}$ are curvature components of the connection $A_{a}^{i}$ and thus quadratic in the canonical variables, but the coefficients $\Gamma_{a}^{i}$ are complicated functions of $E_{i}^{a}$. All these components contain spatial derivatives while time derivatives are replaced by momenta.

To illustrate these variables we consider isotropy as an example. The fields can then be written as $E_{i}^{a}=p \delta_{i}^{a}$ and $A_{a}^{i}=c \delta_{a}^{i}$ with only two remaining canonical variables $|p|=\frac{1}{4} a^{2}$ and $c=\frac{1}{2}(k+\gamma \dot{a})$ where $a$ is the scale factor of a Friedmann-Robertson-Walker metric, $\dot{a}$ its derivative in proper time, and $k=0$ or $k=1$ the curvature parameter which enters

\footnotetext{
${ }^{4}$ The canonical ADM variables [15] for general relativity are defined in terms of the spatial metric $q_{a b}$ and extrinsic curvature $K_{a b}=\frac{1}{2 N}\left(\mathcal{L}_{t} q_{a b}-2 D_{(a} N_{b)}\right)$, where the time function $t$ determines spatial slices $\Sigma_{t}: t=$ const and time is measured along a time evolution vector field $t^{a}=N n^{a}+N^{a}$ decomposed into lapse function $N$ and shift vector $N^{a}$ using the unit normal $n^{a}$ to $\Sigma$.
} 
through the spin connection. (For negative spatial curvature $k=-1$ the expression for the connection is not of the above diagonal form [19].)

The isotropic Hamiltonian constraint simplifies compared to the full expression and is given by

$$
H=-\frac{3}{8 \pi G}\left(\gamma^{-2}(c-k / 2)^{2}+k^{2} / 4\right) \sqrt{|p|}+H_{\text {matter }}(p)=0
$$

with some matter Hamiltonian $H_{\text {matter }}$ Using the transformation of variables above, this can easily be checked to reduce to the Friedmann equation while it generates as Hamiltonian equations of motion the Raychaudhuri equation by $\dot{p}=\{p, H\}, \dot{c}=\{c, H\}$ as well as matter evolution equations if matter fields are present.

To proceed with the full theory, we then have to see how to represent the canonical variables as an operator algebra on a Hilbert space such that Poisson relations become commutator relations. This requires one to smear fields by integrating them over suitable regions, but in a background independent quantization should be done in such a way that no background metric is introduced.

\section{A.2 Holonomies and fluxes}

At this point, we encounter one of the main advantages of Ashtekar variables: they allow a natural smearing of basic fields $\left(A_{a}^{i}, E_{j}^{b}\right)$ to linear objects without introducing a background, while still leading to a well-defined algebra. This comes about because we can naturally integrate a connection along a curve, also taking the path ordered exponential to have good gauge transformation properties under local $\mathrm{SU}(2)$, to give holonomies $h_{e}(A)=\mathcal{P} \exp \int_{e} \tau_{i} A_{a}^{i} \dot{e}^{a} \mathrm{~d} t$ for an arbitrary curve $e$ in the spatial manifold $\Sigma$. Similarly, a densitized vector field can naturally be integrated over 2 -surfaces $S$ to obtain fluxes $F_{S}(E)=\int_{S} \tau^{i} E_{i}^{a} n_{a} \mathrm{~d}^{2} y$. This integration in one plus two dimensions, without introducing any background measure, ${ }^{5}$ turns out to remove all delta functions in the classical Poisson relations and thus results in a well-defined algebra to be represented on a Hilbert space.

Such a representation is the basis for a background independent quantization [20]. One also has to require that the representation one is using is covariant under spatial diffeomorphisms since these transformations have to be removed as gauge. Under this condition, an irreducible, cyclic representation of the holonomy-flux algebra is then uniquely determined [21]. Thus, all states can be obtained from operators acting on a "ground state" in which no geometry at all is excited. One can construct all states in the connection representation, i.e. as functionals of the connection, by using holonomies as multiplication operators. The "ground state" is just a constant on the space of connections, and by multiplication with holonomies one "creates" dependence on the connection along edges. This results in spin

\footnotetext{
${ }^{5}$ The edges $e$ and surfaces $S$ are not fixed but appear as labels, just as points $x$ appear as labels in usual field formulations. Topological and differential background structures still need to be chosen to define the basic objects, but this does not prevent background independence to be realized as in classical general relativity. Also there, one has to choose a topology and differential structure before formulating the field equations, but one does not split the metric into a background plus fields on that background. Background independence in loop quantum gravity is to be understood in the same sense.
} 
network states [25] of the form

$$
T_{g, j, C}(A)=\prod_{v \in g} C_{v} \cdot \prod_{e \in g} \rho_{j_{e}}\left(h_{e}(A)\right)
$$

which are labeled by an oriented graph $g$, irreducible $\mathrm{SU}(2)$ representations $j$ on its edges and gauge invariant contraction matrices $C$ in its vertices. An example is the Wilson loop in the fundamental representation,

$$
W_{g}(A)=\epsilon_{A C} \epsilon^{B D} \cdot\left(h_{1}(A)\right)_{B}^{A}\left(h_{2}(A)\right)_{D}^{C}=\left(h_{1}(A)\right)_{B}^{A}\left(h_{2}(A)^{-1}\right)_{A}^{B}=\operatorname{tr}\left(h_{1}(A) h_{2}(A)^{-1}\right),
$$

for a loop seen as two edges from one vertex to another one oriented in the same way. This gives two holonomies $h_{1}$ and $h_{2}$ between the vertices on which the $\epsilon$ tensors are contraction matrices. In this way, one automatically obtains the trace of a closed holonomy which is gauge invariant, while using other contractions would not give an invariant function. More generally, one can construct gauge invariant spin network states with intersection points and form a basis of all states on connections.

\section{A.3 Discrete geometry}

Fluxes, being conjugate to holonomies, become derivative operators on spin network states acting as

$$
\hat{F}_{S} f_{g}=-8 \pi i \gamma G \hbar \int_{S} \mathrm{~d}^{2} y \tau^{i} n_{a} \frac{\delta f_{g}(h(A))}{\delta A_{a}^{i}(y)}=-8 \pi i \gamma \ell_{\mathrm{P}}^{2} \sum_{e \in g} \int_{S} \mathrm{~d}^{2} y \tau^{i} n_{a} \frac{\delta h_{e}}{\delta A_{a}^{i}(y)} \frac{\mathrm{d} f_{g}(h)}{\mathrm{d} h_{e}} .
$$

Here, the Planck length $\ell_{\mathrm{P}}=\sqrt{G \hbar}$ arises automatically. There are non-zero contributions only if the surface $S$ in the flux intersects edges of the graph $g$ of the state, and individual contributions from intersection points are determined by "angular momentum operators" ( $\mathrm{su}(2)$ derivatives) acting on holonomies. Since such operators have discrete spectra, one sees that fluxes and thus spatial geometry are discrete. Operators such as area and volume can be constructed from fluxes alone and inherit the discreteness of spectra [22]. Zero is always an eigenvalue contained in the spectra, usually of high degeneracy, corresponding to space which is "empty" even of geometry.

While this property indicates discreteness of quantum geometry at least spatially and at the kinematical level, the fact that zero is an eigenvalue in the discrete part of the spectrum also spells trouble: it means that the volume operator, or any of its local contributions corresponding to $\sqrt{\operatorname{det} q}$, does not have a densely defined inverse. However, inverse powers of the determinant of the metric are required for matter Hamiltonians as well as the Hamiltonian constraint. For a scalar field, for instance, we have the classical matter Hamiltonian

$$
H_{\phi}=\int \mathrm{d}^{3} x\left(\frac{1}{2} \frac{p_{\phi}^{2}+E_{i}^{a} E_{i}^{b} \partial_{a} \phi \partial_{b} \phi}{\sqrt{\left|\operatorname{det} E_{j}^{c}\right|}}+\sqrt{\left|\operatorname{det} E_{j}^{c}\right|} V(\phi)\right)
$$


where not only the field variables are to be quantized but also metric components, in particular the inverse determinant, in quantum gravity.

Fortunately, this problem can be solved using identities such as 23 .

$$
\left\{A_{a}^{i}, \int \sqrt{|\operatorname{det} E|} \mathrm{d}^{3} x\right\}=2 \pi \gamma G \epsilon^{i j k} \epsilon_{a b c} \frac{E_{j}^{b} E_{k}^{c}}{\sqrt{|\operatorname{det} E|}}
$$

which allow one to express an inverse power of densitized triad components by a Poisson bracket between connection components and only positive powers of the triad components. The connection components $A_{a}^{i}$, furthermore, can be approximated by holonomies, which is necessary because only holonomies are represented on the Hilbert space. Inserting appropriate holonomies and the volume operator, and replacing the Poisson bracket by $(i \hbar)^{-1}$ times a commutator results in a well-defined operator which has the correct classical limit corresponding to inverse powers of densitized triad components.

Since such operators are densely defined [24, and sometimes even bounded [26], they cannot be identical to an inverse of volume. In particular at small volume scales there are deviations between the classical inverse and quantum behavior. While this modified behavior is not determined uniquely, its characteristic properties are robust. Different versions arise because there are many different ways to re-write inverse triad components, e.g. using different representations for holonomies, which all give the same classical expression but differ in quantum properties contained in their spectra (see [27] for explicit examples). There are thus quantization ambiguities, as always when one quantizes expressions which are non-linear in basic quantities.

\section{A.4 Dynamics}

Inverse powers of densitized triad components are also necessary for the Hamiltonian constraint (24). The same type of modifications thus results. Moreover, we have to express the curvature components $F_{a b}^{i}$ in the first line of (24) in terms of holonomies before we can quantize. This can be done as usually in gauge theories using $s_{1}^{a} s_{2}^{b} F_{a b}^{i} \tau_{i}=\Delta^{-1}\left(h_{\alpha}-1\right)+O(\Delta)$ for a holonomy around a closed loop $\alpha$ of coordinate area $\Delta$ and with unit tangent vectors $s_{1 / 2}$ as in Fig. 11.

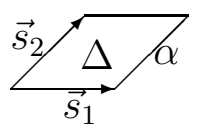

Figure 1: Shape of loops used to express curvature components in terms of holonomies.

The second line of (24), finally, requires us to quantize the spin connection components which are complicated functionals of the triad, and also contain their inverse. In general, it is easier to quantize the combination $K_{a}^{i}=\gamma^{-1}\left(A_{a}^{i}-\Gamma_{a}^{i}\right)$ directly, which can again be written as a Poisson bracket [23]

$$
K_{a}^{i}=\gamma^{-1}\left(A_{a}^{i}-\Gamma_{a}^{i}\right) \propto\left\{A_{a}^{i},\left\{\int \mathrm{d}^{3} x F_{a b}^{i} \frac{E_{j}^{a} E_{k}^{b}}{\sqrt{|\operatorname{det} E|}}, \int \sqrt{|\operatorname{det} E|} \mathrm{d}^{3} x\right\}\right\}
$$


while specific models may allow simpler direct quantizations of spin connection components 28, 29.

Also here, there are several quantization ambiguities and several classes of operators. Any such construction results, understandably, in an operator which is difficult to handle for explicit calculations. It is thus helpful to look at symmetric models at the quantum level [30, which display the characteristic features. Such models, in turn, can then also provide feedback to the full theory in order to guide or specify constructions there. In the simplest models, isotropic ones [31], the basic variables $c$ and $p$ are represented in a loop quantization on orthonormal states $\langle c \mid \mu\rangle=e^{i \mu c / 2}$ with real labels $\mu \in \mathbb{R}[32$. The basic operators then act as $\hat{p}|\mu\rangle=\frac{1}{6} \gamma \ell_{\mathrm{P}}^{2} \mu|\mu\rangle$ and $\widehat{e^{i \mu^{\prime} c / 2}}|\mu\rangle=\left|\mu+\mu^{\prime}\right\rangle$.

Following the construction of Hamiltonian constraint operators gives [31, 28, 32

$$
\hat{H}|\mu\rangle=\frac{3}{16 \pi G \gamma^{3} \ell_{\mathrm{P}}^{2}}\left(V_{\mu+1}-V_{\mu-1}\right)\left(e^{-i k}|\mu+4\rangle-\left(2+k^{2} \gamma^{2}\right)|\mu\rangle+e^{i k}|\mu-4\rangle\right)
$$

where differences in volume eigenvalues $V_{\mu}=\left(\gamma \ell_{\mathrm{P}}^{2}|\mu| / 6\right)^{3 / 2}$ come from commutators, and the shifts in state labels arise from operators $\widehat{e^{i \mu c / 2}}$ used to quantize $c^{2}$ in the classical constraint (25). Expanding difference operators in a Taylor series of differential operators then results in higher order terms, i.e. higher powers of $c$ or $\dot{a}$ but no higher spatial derivatives in homogeneous models, which become important away from semiclassical regimes [33. Similarly, the matter Hamiltonian requires a quantization of $a^{-3}=|p|^{-3 / 2}$, while $\hat{p}$ does not have a densely defined inverse. Re-writing as before results in well-defined expressions which modify the classical behavior on small scales (see [27] for explicit spectra).

\section{A.5 Sources for quantum corrections}

This outline of general constructions shows which types of corrections we have to expect from loop quantum gravity if we use it to derive effective equations from the expectation value of $\hat{H}$. There are

- modified coefficients due to the small-scale behavior of quantized inverse powers of triad components in matter Hamiltonians as well as the Hamiltonian constraint and

- higher order spatial derivatives which arise from replacing local curvature and connection components by holonomies along extended loops; this also implies spatial non-locality.

In addition to that, higher order time derivatives, although not obvious from the loop constructions, result as per our general discussion in the main text. There is no complete derivation yet, but several studies for isolated specific corrections of different origin have been performed. In general, one has to consider all of them, each one to a certain order in perturbative treatments, since they have different magnitudes depending on which regime is being considered. This is similar to usual effective action pictures which contain modified coefficients of different forms and also several higher order and higher derivative corrections. 
Although the derivation of a low energy effective action will be difficult since so far no perturbative vacuum is known, the general procedure of effective systems is applicable. The types of corrections expected from loop quantum gravity are then completely analogous to what one has for quantum field theories on a background. In particular, loop quantum gravity does give rise to quantum corrections to the classical Einstein-Hilbert action.

\section{B Effective actions from string theory}

While it is difficult to define a perturbative vacuum or other state corresponding to Minkowski space in loop quantum gravity, string theory [34 is originally defined perturbatively for string worldsheets in a background spacetime. This formulation lends itself directly to the computation of a low energy effective action. Consistency of the theory then requires the introduction of additional fields which also occur in the effective action coupled to gravity. For gravity itself, higher curvature terms arise. This is often not derived by the usual procedure to arrive at low energy effective actions but by using conditions for the $\beta$-functions to vanish in agreement with the preservation of conformal invariance of the classical theory. This has the advantage of being applicable in different background spacetime geometries.

Low energy effective actions are thus not just obtained around the Minkowski vacuum but there are additional parameters to specify the background geometry. This is in agreement with general expectations formulated in Sec. 固 but does not fully include parameters for true quantum degrees of freedom such as spread. The derivation of such an effective action would require more than perturbative aspects. The available results are thus valid for low energy properties of the theory but not necessarily for aspects of cosmology.

To compare with the results currently available for loop quantum gravity, there is, as often, a very complementary picture: in one case it is difficult to define a non-degenerate vacuum state while the other is built around such a state and describing strong deviations from this state is more involved. Nonetheless, both formulations agree on the types of corrections that are expected in a general picture, although calculations are not yet detailed enough for a quantitative comparison. Coefficients of effective equations as well as covariance properties can be quite different, which will allow interesting means to compare features of both theories.

\section{B.1 Classical Strings}

In order to discuss the different approaches from string theory to the effective field equations, we first introduce the framework briefly based on the standard references [34] and [35] in the field. String theory is based on the premise that there must exist a theory of which general relativity as well as the standard model of particle physics are low energy limits. Although we will not discuss how the standard model might arise, it is important to recall that the different standard model fields of diverse spin, together with the spin two metric field and many new yet unseen fields, are supposedly oscillation modes of a string. 
The string action for a reparametrization invariant 2-dimensional Lorentzian "worldsheet" is given by the Nambu-Goto action

$$
S_{N G}\left[X^{\mu}\right]=-\frac{1}{2 \pi \alpha^{\prime}} \int_{M} \mathrm{~d} \tau \mathrm{d} \sigma \sqrt{-\operatorname{det} h_{\alpha \beta}}
$$

where $h_{\alpha \beta}$ is the worldsheet metric induced by the background metric $G_{\mu \nu}(X)$ of the "target space," a higher dimensional manifold with coordinates $X^{\mu}$ assumed to contain the world as we see it. A canonical quantization of the square root Lagrangian is difficult, but can be avoided by introducing an auxiliary worldsheet metric $\gamma_{\alpha \beta}$ and using the equivalent Polyakov action

$$
S_{P}\left[X^{\mu} ; \gamma_{\alpha \beta}\right]=-\frac{1}{4 \pi \alpha^{\prime}} \int_{M} \mathrm{~d} \tau \mathrm{d} \sigma \sqrt{-\operatorname{det} \gamma_{\delta \epsilon}} \gamma^{\alpha \beta} G_{\mu \nu} \partial_{\alpha} X^{\mu} \partial_{\beta} X^{\nu}
$$

In a Minkowskian target space, $G_{\mu \nu}(X)=\eta_{\mu \nu}$, the equations of motion are obtained by varying the Polyakov action with respect to $X^{\mu}$, thus obtaining

$$
\partial_{\alpha} \sqrt{|\gamma|} \gamma^{\alpha \beta} \partial_{\beta} X^{\mu}(\tau, \sigma)=0
$$

Solutions can be constructed once boundary conditions are specified, for which there are periodic ones (closed string) or of Dirichlet, Neumann or even mixed type where the positions of endpoints or their derivatives are fixed. It is usual to fix all the gauge freedom in the worldsheet metric and keep the conformal transformations as the only degree of freedom left, $\gamma_{\alpha \beta}(\tau, \sigma)=\omega^{2}(\tau, \sigma) \eta_{\alpha \beta}$. In this conformal gauge, the equations are manifestly invariant under changes of the conformal factor $\omega$. In order to keep track of the physical degrees of freedom of the theory, it is sometimes more convenient to choose the so called light cone gauge where $X^{0}(\tau, \sigma)+X^{1}(\tau, \sigma)=\tau$ and $X^{0}-X^{1}=X^{-}$. If a proper coordinate transformation on the worldsheet is performed, it is possible to write the Polyakov action in the following shape

$$
S_{p p}=-\frac{1}{2 \pi \alpha^{\prime}} \int \mathrm{d} \tau \int_{0}^{\ell} \mathrm{d} \sigma\left[2 \gamma_{\sigma \sigma} \partial_{\tau} X^{-}+\gamma_{\sigma \sigma} \partial_{\tau} X^{i} \partial_{\tau} X^{i}-\gamma_{\sigma \sigma}^{-1} \partial_{\sigma} X^{i} \partial_{\sigma} X^{i}\right]
$$

which for Neumann boundary conditions can be solved by

$$
X^{i}(\tau, \sigma)=x^{i}+\frac{p^{i}}{p^{+}} \tau+i \sqrt{2 \alpha^{\prime}} \sum_{0 \neq n=-\infty}^{\infty} \frac{\alpha_{n}^{i}}{n} \cos \left(\frac{\pi n \sigma}{\ell}\right) e^{-\frac{i}{\ell} \pi n c \tau} .
$$

where $i \neq 0,1$ and $p^{+}$is the average of the momentum canonically conjugate to $X^{-}$. For other boundary conditions, one obtains analogous situations with solutions decomposed in mode expansions where the amplitude $\alpha_{n}^{i}$ of each mode is an independent degree of freedom, with Poisson brackets $\left\{\alpha_{m}^{i}, \alpha_{-n}^{j}\right\}=-i m \delta^{i j} \delta_{m n}$. 


\section{B.2 Quantum Strings}

The classical Lorentzian and conformal symmetries of string theory are not necessarily preserved after quantization. This is only the case if the target space is 26-dimensional (or 10-dimensional for supersymmetric versions). In particular conformal symmetry is the cornerstone of string theory and its consistency as a physical theory depends on it. It is also crucial as the source of many mathematical tools which may provide the calculational power that a good physical theory should be equipped with.

Exact quantization can be performed only in special cases where the string action becomes a free theory on the worldsheet. This usually requires a background metric of maximal symmetry among which Minkowski and anti-de Sitter spacetimes have been most widely studied (see [36] for general references on strings in background fields). The modes $\alpha_{n}^{i}$ of the classical string are then turned into operators, satisfying the Heisenberg algebra $\left[\hat{\alpha}_{m}^{i}, \hat{\alpha}_{-n}^{j}\right]=m \delta^{i j} \delta_{m n}$ where the reality condition of $X^{\mu}$ requires $\left(\hat{\alpha}_{-n}^{j}\right)^{\dagger}=\hat{\alpha}_{n}^{j}$. This operator can be considered as a creation operator for the mode of rotation labeled by $n$ corresponding to the spin of the created particle.

In addition, there are constraints which, when quantized, obey a Virasoro algebra and annihilate physical states. The Hamiltonian then determines the mass spectrum, and is for the open string given by

$$
H=\frac{1}{2 p^{+}} p^{i} p^{i}+\frac{1}{2 p^{+} \alpha^{\prime}}\left(\sum_{n>0} \alpha_{-n}^{i} \alpha_{n}^{i}+A\right)
$$

where regularization yields $A=\frac{2-D}{24}$ with $D$ the target space dimension. Therefore, the lowest mass state can be constructed as $|0 ; k\rangle$ with squared mass $m^{2}=\frac{2-D}{24 \alpha^{\prime}}$ and wave vector $k$. The vectorial mode $\alpha_{-1}^{i}|0 ; k\rangle$ has $m^{2}=\frac{26-D}{24 \alpha^{\prime}}$, and higher spin excitations have larger masses.

It has been argued that a theory including exclusively open string modes is inconsistent, introducing closed string modes

$$
X^{i}(\tau, \sigma)=x^{i}+\frac{p^{i}}{p^{+}} \tau+i \sqrt{\frac{\alpha^{\prime}}{2}} \sum_{0 \neq n=-\infty}^{\infty}\left(\frac{\bar{\alpha}_{n}^{i}}{n} e^{-\frac{2 \pi i n}{\ell}(\sigma+c \tau)}+\frac{\tilde{\alpha}_{n}^{i}}{n} e^{\frac{2 \pi i n}{\ell}(\sigma-c \tau)}\right) .
$$

Now, there is a constraint that removes any state with different numbers of bar and tilde operators from the physical spectrum. The lowest mass states are then for the scalar degree of freedom $|\overline{0}, \tilde{0} ; k\rangle$ with squared mass $m^{2}=\frac{2-D}{6 \alpha^{\prime}}$ and the state $\bar{\alpha}_{-1}^{i} \tilde{\alpha}_{-1}^{j}|\overline{0}, \tilde{0} ; k\rangle$ with $m^{2}=\frac{26-D}{6 \alpha^{\prime}}$ can be decomposed into the $S O(D-2)$ fundamental representations in the tensor product of two fundamental ones.

Since self-consistency of the theory requires $D=26$, the fundamental scalars are tachyons and the vector excitation, the antisymmetric tensor, symmetric traceless tensor and trace part would all become massless fields, usually regarded as a $U(1)$ connection, a fundamental antisymmetric tensor ( $B$-Field), the graviton field and a dilaton scalar field. Tachyonic states are absent in supersymmetric versions, while the other excitations remain. For our purposes of illustrating effective corrections, we focus on the propagation of the massless sector of the theory. 


\section{B.3 Effective Field equations}

Since the squared mass spectrum is inversely proportional to the small $\alpha^{\prime}$, the only relevant particles in the low energy effective dynamics of the string are the massless ones. It is therefore of interest to construct field equations which describe the propagation of the string modes individuallyand, simultaneously, introduce interactions between the effective fields at an effective level as they follow from scattering amplitudes derived from the fundamental string processes. It turns out that self interactions (4-point functions) of symmetric tensor modes correspond to those coming from the linearized Einstein equations. Thus, very near to 26-dimensional Minkowski spacetime the massless symmetric tensor excitations of the closed string behave as gravitational perturbations.

Field equations for each separate string mode can be considered as the linearized expressions, corresponding to two point functions. For higher order terms in the expansion, it is then necessary to compute higher point functions which is more difficult. In fact, relevant perturbative expansions have been carried out in the $\alpha^{\prime}$ expansion as well as in genus of the string worldsheet topology, but most notorious is the inclusion of effects due to the presence of highly massive states. Their effects are included by new interaction terms, similarly to a Fermi interaction. Obviating the need for other fields, an ansatz for an effective (low energy) action can be considered as a sum of invariants constructed from different powers of the Riemann tensor with free coefficients. Based on properties of the different string theory models, these coefficients in the low energy action can then be determined.

Parallel results have been performed based on conformal field theory techniques. These are very powerful and allow the study of the unperturbed equations through the use of operator product expansions, conformal invariance, the Virasoro algebra and vertex operators. In order to obtain physical predictions one deals with the $N$ particles scattering amplitude obtained by an $S$-matrix. In these terms, it becomes very useful to proceed by substituting the problem by a sum over all possible configurations through the Feynman integral. In this context it is possible to obtain an alternative viewpoint of the pathology that arises in the scattering amplitudes which in the lightcone gauge appears as an anomaly in the Lorentz invariance unless $D=26$. In the present formalism the anomaly appears in the conformal

symmetry, which at the classical level was realized as $\gamma^{\alpha \beta} \frac{\delta}{\delta \gamma^{\alpha \beta}} S_{P}\left[X^{\mu}\right]=\sqrt{\gamma} T_{\alpha}^{\alpha}=0$ but holds no longer after quantization.

As a matter of fact, the scale invariance is evident due to the fact that the Lagrangian contains only dimensionless coupling constants. In spite of that, after quantization one obtains nonzero beta functions which govern the flow of the parameters of the theory according to the renormalization group equations. The trace of the energy momentum tensor then does not vanish after quantizing, and in fact is proportional to the beta functions. They thus have to be set to zero in order to make the results of physical predictions independent of the fiducial metric $\gamma_{\alpha \beta}$.

Conformal field theory tools allow the computation of $\beta$ functions associated to each of the fields. For the gravitation mode, the result is proportional to Einstein's equations. Therefore, requiring that we are in the physical sector of string theory by simply imposing that conformal invariance is preserved is equivalent to imposing Einstein's equations. These 
beta functions can in fact be corrected to higher order in $\alpha^{\prime}$ or in the genus expansion, thus ideally leading to effective actions with higher powers of the invariants of the theory such as $R^{n}$ although the results depend on the type of string model.

\section{B.4 Free parameters}

From this brief review it is clear that techniques of string theory are most suitable to low energy effective actions, but make it more complicated to derive more general effective systems. Parameters specifying states to expand around, as they occur in the general case, will thus also be present for string theory, although current technology does not allow one to include them. Some of those parameters can be included by choosing different backgrounds, but this does not include truly quantum variables. One can see directly how these parameters are excluded by presently available calculations: As we noted before, a general effective system will not be manifestly invariant under all symmetries of the quantum theory because one has to choose a state which may not be invariant. Similarly, setting $\beta$-functions to zero implicitly selects special states used for an effective system. This removes most of the freedom in choosing states, similarly to picking explicitly the vacuum state for a low energy effective action which preserves all symmetries manifestly.

\section{References}

[1] H. Euler and W. Heisenberg, Consequences of Dirac's Theory of the Positron, Z. Phys. 98 (1936), 714, physics/0605038, J. Schwinger, On Gauge Invariance and Vacuum Polarization, Phys. Rev. 82 (1951), 664-679.

[2] L. Dolan and R. Jackiw, Gauge-invariant signal for gauge-symmetry breaking, Phys. Rev. D 9 (1974), 2904-2912; F. Cametti, G. Jona-Lasinio, C. Presilla, and F. Toninelli, Comparison between quantum and classical dynamics in the effective action formalism, In Proceedings of the International School of Physics "Enrico Fermi", Course CXLIII, 431-448, IOS Press, Amsterdam, 2000, quant-ph/9910065.

[3] X. Jaén, J. Llosa, and A. Molina, A reduction of order two for infinite-order Lagrangians, Phys. Rev. D 34 (1986), 2302-2311.

[4] M. Bojowald, H. Morales-Técotl, and H. Sahlmann, On Loop Quantum Gravity Phenomenology and the Issue of Lorentz Invariance, Phys. Rev. D 71 (2005), 084012, gr-qc/0411101.

[5] J. Z. Simon, Higher-derivative Lagrangians, nonlocality, problems, and solutions, Phys. Rev. D 41 (1990), 3720-3733.

[6] M. Bojowald and A. Skirzewski, Effective Equations of Motion for Quantum Systems, math-ph/0511043. 
[7] T. W. B. Kibble, Geometrization of quantum mechanics, Commun. Math. Phys. 65 (1979), 189-201; A. Heslot, Quantum mechanics as a classical theory, Phys. Rev. D 31 (1985), 1341-1348

[8] A. Ashtekar and T. A. Schilling, Geometrical Formulation of Quantum Mechanics, In On Einstein's Path: Essays in Honor of Engelbert Schücking, 23-65, Springer, New York, 1999, gr-qc/9706069

[9] J. Willis, On the Low-Energy Ramifications and a Mathematical Extension of Loop Quantum Gravity, PhD thesis, The Pennsylvania State University, 2004.

[10] N. C. Dias, A. Mikovic, and J. N. Prata, Coherent States Expectation Values as Semiclassical Trajectories, hep-th/0507255.

[11] R. Jackiw and A. Kerman, Time Dependent Variational Principle And The Effective Action, Phys. Lett. A 71 (1979) 158-162

[12] M. Bojowald and A. Skirzewski, Effective theory for the cosmological generation of structure, in preparation.

[13] R. Gambini and J. Pullin, Nonstandard optics from quantum spacetime, Phys. Rev. D 59 (1999), 124021, gr-qc/9809038 J. Alfaro, H. A. Morales-Técotl, and L. F. Urrutia, Quantum gravity corrections to neutrino propagation, Phys. Rev. Lett. 84 (2000), 2318-2321, gr-qc/9909079; J. Alfaro, H. A. Morales-Técotl, and L. F. Urrutia, Loop quantum gravity and light propagation, Phys. Rev. D 65 (2002), 103509, hep-th/0108061.

[14] C. Rovelli, Quantum Gravity, Cambridge University Press, Cambridge, UK, 2004; T. Thiemann, Introduction to Modern Canonical Quantum General Relativity, gr-qc/0110034 A. Ashtekar and J. Lewandowski, Background independent quantum gravity: A status report, Class. Quantum Grav. 21 (2004), R53-R152, gr-qc/0404018; M. Bojowald, Loop Quantum Cosmology, Living Rev. Relativity 8 (2005), 11, gr-qc/0601085, http://relativity.livingreviews.org/Articles/lrr-2005-11/.

[15] R. Arnowitt, S. Deser, and C. W. Misner, The Dynamics of General Relativity, Wiley, New York, 1962.

[16] A. Ashtekar, New Hamiltonian Formulation of General Relativity, Phys. Rev. D 36 (1987), 1587-1602.

[17] J. F. Barbero G., Real Ashtekar Variables for Lorentzian Signature Space-Times, Phys. Rev. D 51 (1995), 5507-5510, gr-qc/9410014.

[18] G. Immirzi, Real and Complex Connections for Canonical Gravity, Class. Quantum Grav. 14 (1997), L177-L181. 
[19] K. Vandersloot, Loop Quantum Cosmology, PhD Thesis, The Pennsylvania State University, 2006.

[20] C. Rovelli and L. Smolin, Loop Space Representation of Quantum General Relativity, Nucl. Phys. B 331 (1990), 80-152.

[21] J. Lewandowski, A. Okołów, H. Sahlmann, and T. Thieman, Uniqueness of diffeomorphism invariant states on holonomy-flux algebras, gr-qc/0504147; C. Fleischhack, Representations of the Weyl Algebra in Quantum Geometry, math-ph/0407006.

[22] C. Rovelli and L. Smolin, Discreteness of Area and Volume in Quantum Gravity, Nucl. Phys. B 442 (1995), 593-619, gr-qc/9411005, Erratum: Nucl. Phys. B 456 (1995) 753; A. Ashtekar and J. Lewandowski, Quantum Theory of Geometry I: Area Operators, Class. Quantum Grav. 14 (1997), A55-A82, gr-qc/9602046; A. Ashtekar and J. Lewandowski, Quantum Theory of Geometry II: Volume Operators, Adv. Theor. Math. Phys. 1 (1997), 388-429, gr-qc/9711031.

[23] T. Thiemann, Quantum Spin Dynamics (QSD), Class. Quantum Grav. 15 (1998), 839-873, gr-qc/9606089.

[24] T. Thiemann, QSD V: Quantum Gravity as the Natural Regulator of Matter Quantum Field Theories, Class. Quantum Grav. 15 (1998), 1281-1314, gr-qc/9705019.

[25] C. Rovelli and L. Smolin, Spin Networks and Quantum Gravity, Phys. Rev. D 52 (1995), 5743-5759.

[26] M. Bojowald, Inverse Scale Factor in Isotropic Quantum Geometry, Phys. Rev. D 64 (2001), 084018, gr-qc/0105067.

[27] M. Bojowald, Quantization ambiguities in isotropic quantum geometry, Class. Quantum Grav. 19 (2002), 5113-5130, gr-qc/0206053 Loop Quantum Cosmology: Recent Progress, In Proceedings of the International Conference on Gravitation and Cosmology (ICGC 2004), Pramana 63 (2004), 765-776, gr-qc/0402053.

[28] M. Bojowald and K. Vandersloot, Loop quantum cosmology, boundary proposals, and inflation, Phys. Rev. D 67 (2003), 124023, gr-qc/0303072.

[29] M. Bojowald, G. Date, and K. Vandersloot, Homogeneous loop quantum cosmology: The role of the spin connection, Class. Quantum Grav. 21 (2004), 1253-1278, gr-qc/0311004; M. Bojowald and R. Swiderski, Spherically Symmetric Quantum Geometry: Hamiltonian Constraint, Class. Quantum Grav. 23 (2006), 2129-2154, gr-qc/0511108.

[30] M. Bojowald and H. A. Kastrup, Symmetry Reduction for Quantized Diffeomorphism Invariant Theories of Connections, Class. Quantum Grav. 17 (2000), 3009-3043, hep-th/9907042. 
[31] M. Bojowald, Isotropic Loop Quantum Cosmology, Class. Quantum Grav. 19 (2002), $2717-2741$, gr-qc/0202077.

[32] A. Ashtekar, M. Bojowald, and J. Lewandowski, Mathematical structure of loop quantum cosmology, Adv. Theor. Math. Phys. 7 (2003), 233-268, gr-qc/0304074.

[33] M. Bojowald, The Semiclassical Limit of Loop Quantum Cosmology, Class. Quantum Grav. 18 (2001), L109-L116, gr-qc/0105113 G. Date and G. Hossain, Effective Hamiltonian for Isotropic Loop Quantum Cosmology, Class. Quantum Grav. 21 (2004), 4941-4953, gr-qc/0407073 M. Bojowald, P. Singh, and A. Skirzewski, Coordinate time dependence in quantum gravity, Phys. Rev. D 70 (2004), 124022, gr-qc/0408094 P. Singh and K. Vandersloot, Semi-classical States, Effective Dynamics and Classical Emergence in Loop Quantum Cosmology, Phys. Rev. D 72 (2005), 084004, gr-qc/0507029

[34] J. Polchinski, String Theory, volume I and II, Cambridge University Press, 1998.

[35] M. B. Green, J. H. Schwarz and E. Witten, Superstring theory, Cambridge University Press, 1987.

[36] C. G. Callan, E. J. Friedan, E. J. Martinec and M. J. Perry, Strings In Background Fields, Nucl. Phys. B262 (1985), 593; C. G. Callan and L. Thorlacius, Sigma Models And String Theory, in Particles, strings and supernovae : proceedings, World Scientific, 1989; A. A. Tseytlin, On The Renormalization Group Approach To String Equations Of Motion, Int. J. Mod. Phys. A 4 (1989), 4249. 